\title{
Descripción de la infraestructura cultural y recreativa en la ciudad del Distrito Central de Honduras
}

Celina Michelle Sosa Caballero

\section{Resumen}

Para poder realizar el estudio Distribución de los Espacios Públicos en el Distrito Central de Honduras, para el Análisis del Equilibrio Territorial de la Oferta Cultural y Recreativa de la Ciudad. En un primer momento se realizó un proceso de levantamiento de datos sobre los distintos tipos de infraestructura cultural y recreativa, cuyo resultado se presenta en la "Descripción de la Infraestructura Cultural y Recreativa en la Ciudad del Distrito Central de Honduras".

Metodológicamente, a partir de la literatura revisada se identificaron las categorías de clasificación de la infraestructura de espacios públicos de la ciudad del Distrito Central, consultando diferentes fuentes de información se generó una base de datos con un total de 365 registros clasificados en 13 categorías y se elaboraron 8 mapas, con los cuales se describen el tipo de infraestructura registrada.

A partir de la Descripción de la Infraestructura Cultural y Recreativa en la Ciudad del Distrito Central de Honduras, se concluye que la mayor parte de las instalaciones son deportivas y poca dotación de instalaciones culturales, además las tendencias de ubicación de instalaciones deportivas se distribuyen por toda el área urbana mientras que las instalaciones culturales se concentran en torno al centro histórico de la ciudad

Palabras clave: Distribución Espacial, Espacios Públicos, Infraestructura cultural, Infraestructura Recreativa.

\section{Abstract}

To make the study Distribution of Public Spaces in the Central District of Honduras, for the Analysis of Territorial Balance of the Cultural and Recreational Offer of the City. Initially was done the process of collection of data on the different types of infrastructure which is presented in the Description of Cultural and Recreational Infrastructure in the city of Distrito Central of Honduras. 
To make this objective, methodologically: from the literature reviewed was identification the classification categories of the infrastructure of public spaces in the city of Distrito Central; consulting different information sources, it was generate a database with the classification of the infrastructure with a total of 365 files and 13 categories, and 8 maps which describes the type of infrastructure recorded.

From the description of the cultural and recreational infrastructure in the city of Distrito Central of Honduras concludes that most of the facilities are sports and little provision of cultural facilities, as well as trends in location of sports facilities are distributed throughout the urban area while cultural facilities are concentrated around the historic city center

Keywords: Spatial Distribution, Public Spaces, Cultural infrastructure, Recreational Infrastructure.

Celina Michelle Sosa Caballero Departamento de Ciencia y Tecnologías de la Información Geográfica, Facultad de Ciencias Espaciales, Universidad Nacional Autónoma de Honduras (UNAH). 


\section{Introducción}

Con la Descripción de la Infraestructura Cultural y Recreativa en la Ciudad del Distrito Central de Honduras, se busca identificar la localización y tipo de infraestructura cultural y recreativa de la ciudad.

En la Agenda 21 Cultural (aprobada en mayo del 2004 por ciudades y gobiernos locales de todo el mundo), en el tema de cultura, sostenibilidad y territorio se define los espacios públicos como: "espacios de cultura". Así mismo, en la Agenda de Ordenamiento Territorial del Concejo Centroamericano de Vivienda y Asentamientos Humanos -CCVAH- (2010), entre los principios, se contempla la calidad del espacio público: "los espacios públicos son elementos esenciales para la vida, dinamismo, identidad y cultura de un asentamiento humano, razón por lo que estos deben ser diseñados y ubicados de forma que respondan a estas funciones. Deben articularse funcionalmente con la vivienda entre otros espacios, integrándose de tal forma que no se conviertan en lugares propicios para afectar la seguridad ciudadana" (CCVAH, 2010. pág. 16).

Partiendo de los principios de que "la diversidad cultural es tan necesaria para la humanidad como la biodiversidad para la naturaleza; la diversidad de las expresiones culturales comporta riqueza y la importancia de un ecosistema cultural amplio, con diversidad de orígenes, agentes, contenidos y diálogo, convivencia e interculturalidad como principios básicos de la dinámica de relaciones ciudadanas" (Ciudades y Gobiernos Locales Unidos, 2004. Pág. 5), contar con un análisis del equipamiento de la oferta cultural y recreativa del Distrito Central es un paso importante para mejorar las condiciones de habitabilidad urbana para el desarrollo personal de los ciudadanos.

En la literatura revisada se han identificado varias definiciones sobre espacios públicos, la más general de ellas los define como: "los espacios públicos son bienes colectivos que pertenecen a todos los ciudadanos. Ningún individuo 0 grupo puede verse privado de su libre utilización, dentro del respeto a las normas adoptadas en cada ciudad" (Ciudades y Gobiernos Locales Unidos, 2004. Pág. 9), de la que se destaca el principio de bien público de estos espacios.

\section{Metodología}

A partir de la literatura revisada, se identificaron las categorías de clasificación de la infraestructura de espacios públicos de la ciudad del Distrito Central; las fuentes consultadas son: Sistema Nacional de Cultura, Informe Honduras, de 
la Organización de Estados Iberoamericanos; un croquis, Mapa de Tegucigalpa sitios de interés del Instituto Hondureños de Turismo (sin fecha, ni escala); Informe de Actividades 2010, Presupuesto y Plan de Trabajo - 2011 del Departamento de Deportes del AMDC; Google Earth; Google Mapas y visitas de campo.

Con la información obtenida en estas fuentes se creó la base de datos que contiene 13 categorías y un total de 365 registros. Esta base de datos contiene los datos geoespaciales, representando los puntos de las coordenadas $X, Y$ de cada una las infraestructuras identificadas.

Por medio de software especializado en sistemas de información geográfica se hizo la localización de la infraestructura cultural y recreativa de la Ciudad.

\section{Resultados}

Los resultados encontrados se han analizado en dos categorías, la primera, característica y distribución de los espacios públicos, donde se identifican el tipo de instalaciones de la infraestructura de espacios públicos y la segunda, la localización espacial de esta infraestructura.

\section{Características y distribución espacial de los espacios públicos de la ciudad del Distrito Central de Honduras}

\section{Tipos de Instalaciones de la Infraestructura de Espacios Públicos}

Los tipos de instalaciones de infraestructura de espacios públicos identificados en el Distrito Central son un total de 365 instalaciones, donde el $31.51 \%$ corresponde a canchas de baloncesto, el $22.74 \%$ corresponde a canchas de fútbol de colonia o barrio, el $17.26 \%$ corresponde a canchas de futbolito privadas, las instalaciones con valores relativos más bajos corresponden a canchas de béisbol, complejos deportivos, estadios y teatros (ver tabla 1). 


\begin{tabular}{|l|r|r|}
\hline Tipo de Infraestructura & Absoluto & Relativo \\
\hline Bibliotecas & 15 & 4.11 \\
\hline Canchas de Fútbol de Colonia o Barrio & 83 & 22.74 \\
\hline Canchas de Futbolito Privadas & 63 & 17.26 \\
\hline Canchas de Béisbol & 2 & 0.55 \\
\hline Canchas de Baloncesto & 115 & 31.51 \\
\hline Canchas Privadas & 22 & 6.03 \\
\hline Cines & 7 & 1.92 \\
\hline Complejos Deportivos & 3 & 0.82 \\
\hline Estadios & 3 & 0.82 \\
\hline Museos & 13 & 3.56 \\
\hline Parques & 31 & 8.49 \\
\hline Areas Protegidas & 3 & 0.82 \\
\hline Teatros & 5 & 1.37 \\
\hline Total & 365 & 100.00 \\
\hline Fuente: Elaboración Propia de Base de datos Marzo - Agosto 2013 & & \\
\hline
\end{tabular}

Tabla 1: Instalaciones del Equipamiento de la Oferta Cultural y

Recreativa de la Ciudad del Distrito Central de Honduras

\section{Localización espacial de la infraestructura de espacios públicos de la ciudad del} Distrito Central de Honduras

Se ha elaborado 8 mapas para representar la infraestructura según categorías, las cuales son: cancha de fútbol de colonias o barrios; canchas de futbolito privadas; canchas de baloncesto; canchas de béisbol, complejos deportivos, estadios; museos, cines, teatros, bibliotecas; canchas privadas y, parques y áreas protegidas.

\section{Cancha de Fútbol de Colonias o Barrios}

La categoría de cancha de fútbol de barrio o colonia, son las que se encuentran localizadas en las diferentes colonias o barrios de la ciudad, generalmente estas canchas son de tierra, señaladas con cal o aserrín, cuyo mantenimiento está bajo la responsabilidad del patronato del barrio y las ligas, en éstas se juegan los campeonatos entre los equipos de barrios y colonias. Las 83 canchas se encuentran dispersas por toda el área urbana (ver mapa 1). 


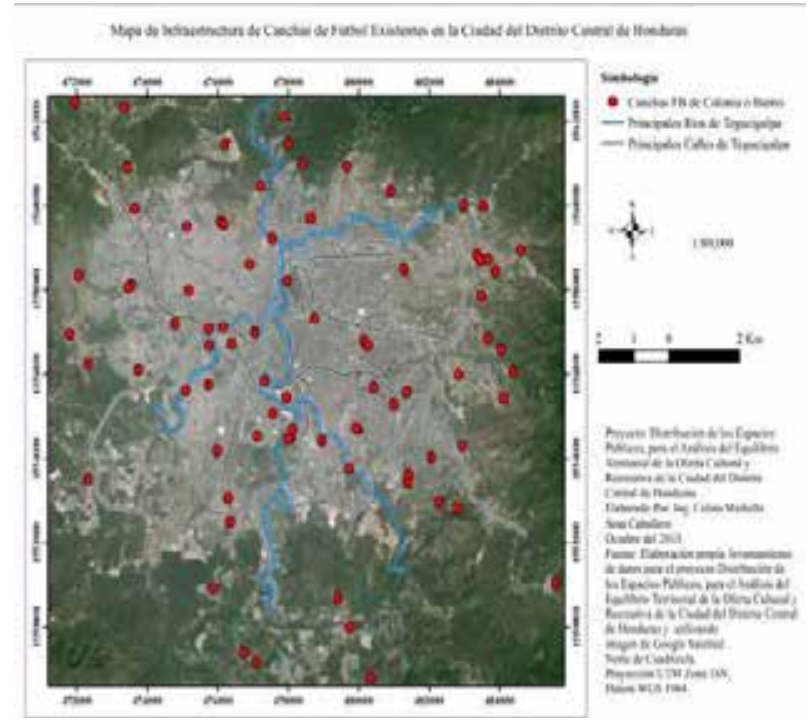

Mapa1. Infraestructura de Canchas de Fútbol Existentes en la Ciudad del Distrito Central

\section{Canchas de Futbolito Privadas}

En estas canchas, los equipos que desean jugar futbol, alquilan las instalaciones por un determinado valor (entre 300 a 2000 Lempiras). Las 63 canchas se encuentran distribuidas por toda el área urbana (Ver Mapa 2).

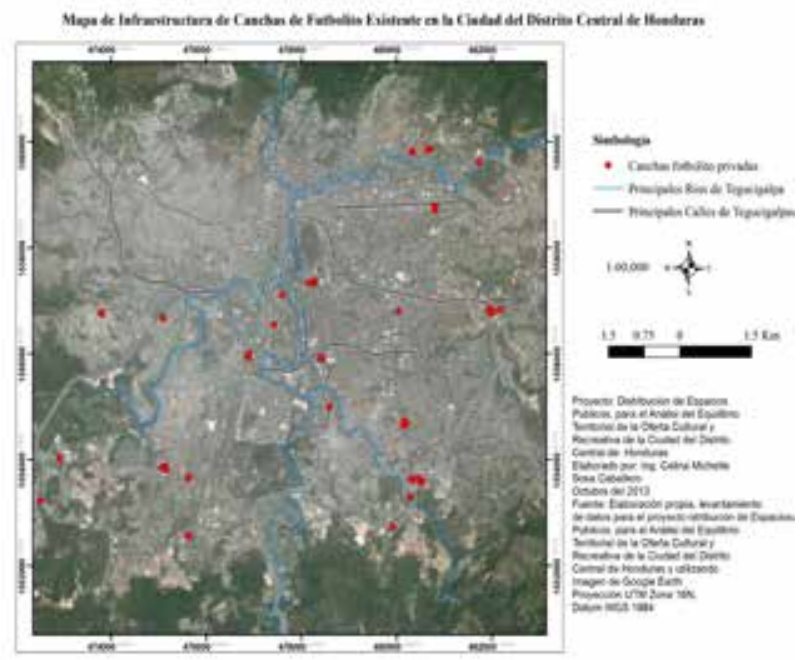

Mapa 2. Infraestructura de Canchas de Futbolito Existentes en la Ciudad del Distrito Central 


\section{Canchas de Baloncesto}

Estas se localizan en las colonias y barrios de la ciudad. Las 115 canchas se encuentran dispersas en el área urbana de la ciudad (Ver Mapa 3).

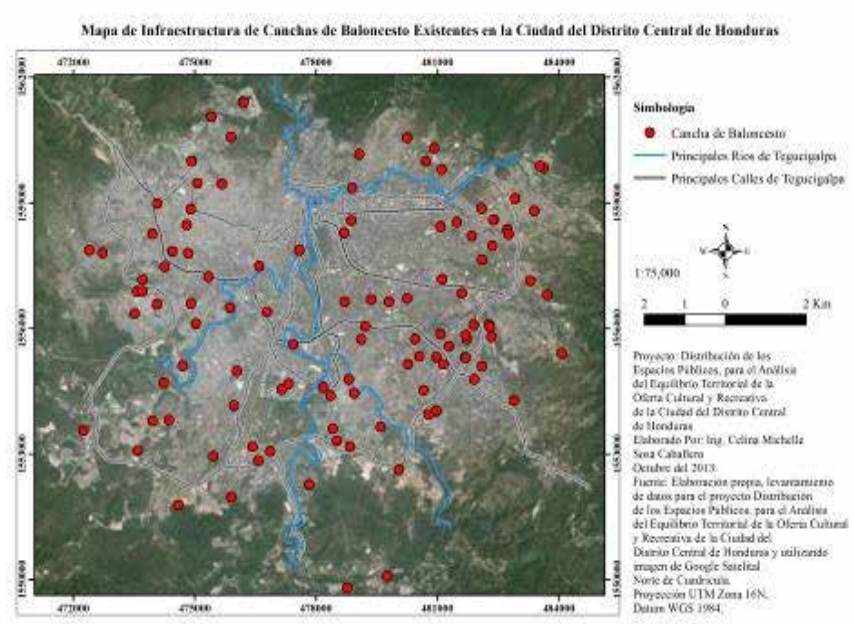

Mapa 3. Infraestructura de Baloncesto Existente en la Ciudad del Distrito Central de Honduras.

\section{Infraestructura Deportiva}

En ésta se han agrupado, los estadios (3), canchas de béisbol (2) y complejos deportivos (3). Los estadios son las instalaciones que cumplen con las normas establecida por la FIFA para jugar al fútbol profesional y los complejos deportivos como La Villa Olímpica y El Palacio Universitario de las Ciencias de la Cultura Física, cuentan con todas las disciplinas de juegos olímpicos para Centro América y el Nacional de Ingenieros, solo con el baloncesto y el Voleibol (ver Mapa 4). 


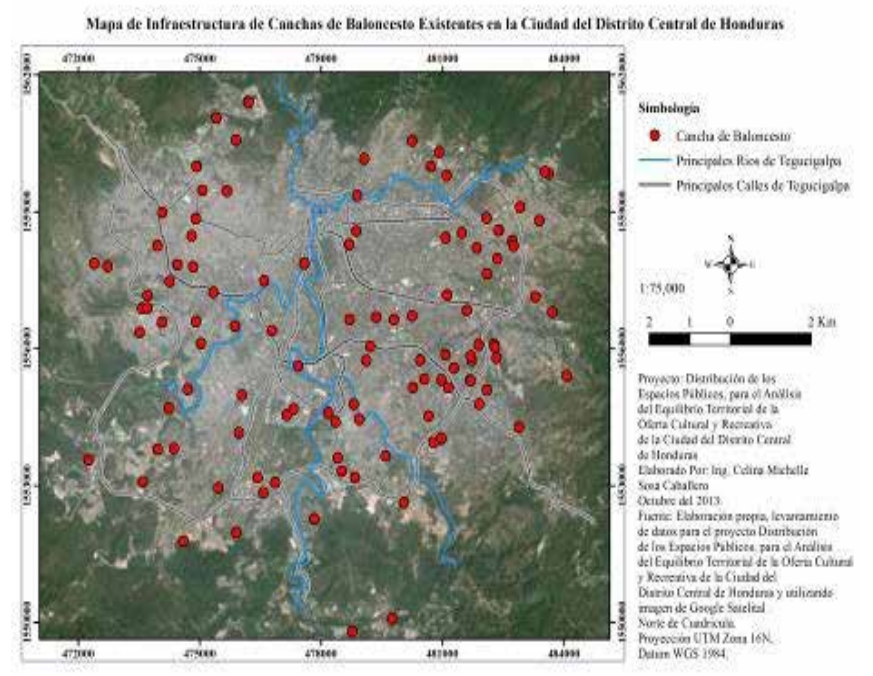

Mapa 4. Infraestructura Deportivos Existentes en la Ciudad del Distrito Central

\section{Infraestructura Cultural}

Estas instalaciones se han agrupado en museos (13), teatros (5), bibliotecas (15) y cines (7), ya que corresponden a la categoría de espectáculo y la cultura.

La mayoría de los museos se localizan en el centro histórico de la ciudad, a excepción de los museos de Bancatlan y Chiminke que se localizan en la zona sureste de la ciudad y el Museo del Aire, en la zona suroeste.

La mayoría de los teatros se encuentran localizados en el centro de la ciudad a excepción del Teatro Renacimiento, localizado en la zona suroeste y El Teatro Nova Centro, en la zona noreste.

Algunas de las bibliotecas se encuentran en el centro y otras dispersas en el resto del área urbana. Los cines se encuentran en los centros comerciales y están fuera del centro histórico (ver mapa 5). 


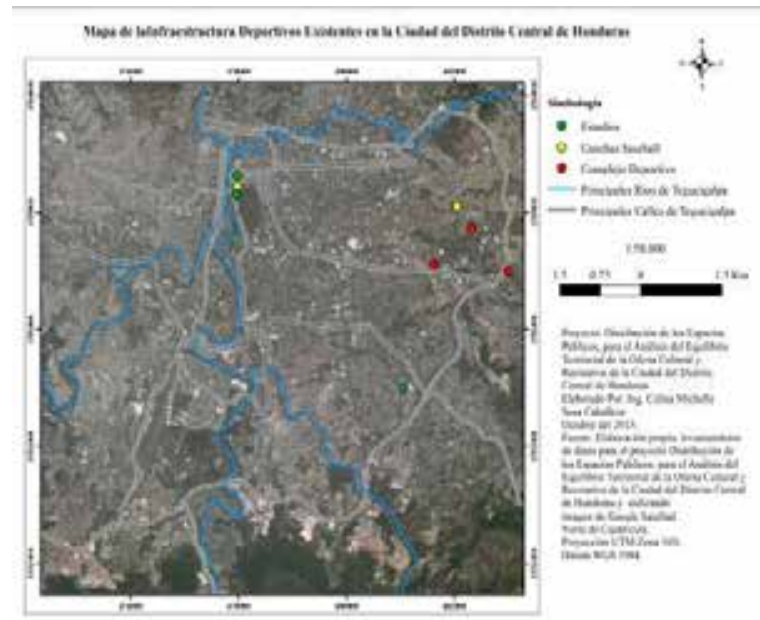

Mapa 5. Infraestructura Cultural Existente en la Ciudad del Distrito Central de Honduras

\section{Canchas Privadas}

Las canchas privadas son las de baloncesto, béisbol y futbol que se encuentran en los clubs privados y colegios profesionales. Las 22 canchas se encuentran dispersas por la ciudad (ver mapa 6).

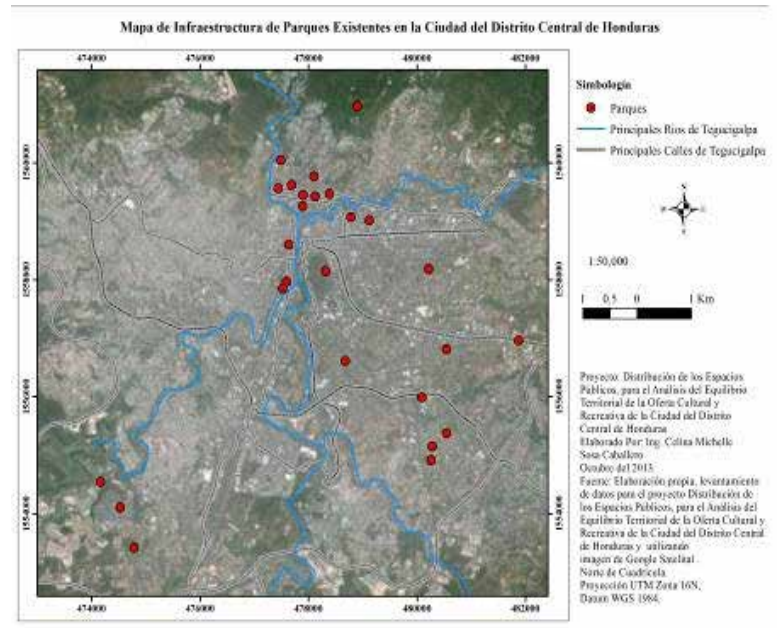

Mapa 6. Infraestructura de Canchas Privadas Existentes en la Ciudad del Distrito Central de Honduras 


\section{Parques y Áreas Protegidas}

Las instalaciones de los parques son las áreas de recreación al aire libre, la mayoría se encuentran localizados en el centro de la ciudad, a excepción de unos pocos que están ubicados en las colonias. Siendo éste, uno de los principales déficit de la ciudad (ver mapa 7).

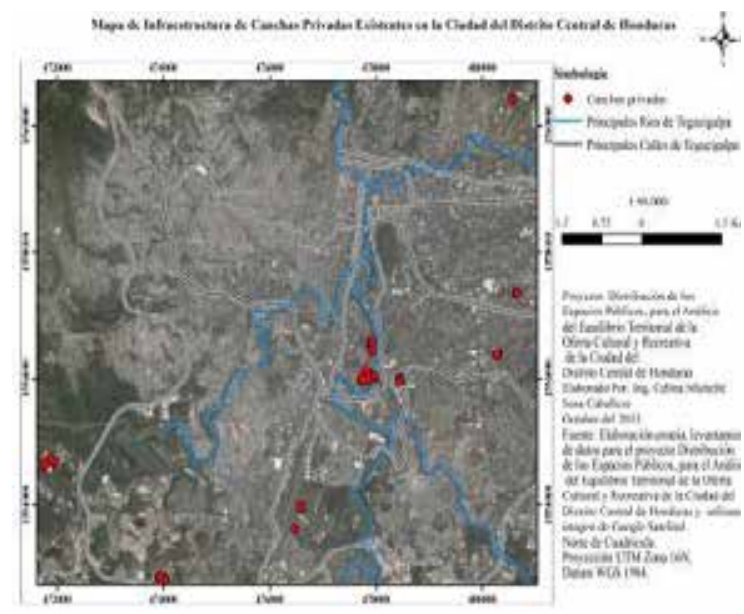

Mapa 7. Infraestructura de Parques Existentes en la Ciudad del Distrito Central de Honduras

Las áreas protegidas son declaradas por el Congreso Nacional, la Tigra y Yerba Buena son protegidas como fuente productoras de agua y El Uyuca como una reserva biológica (ver mapa 8).

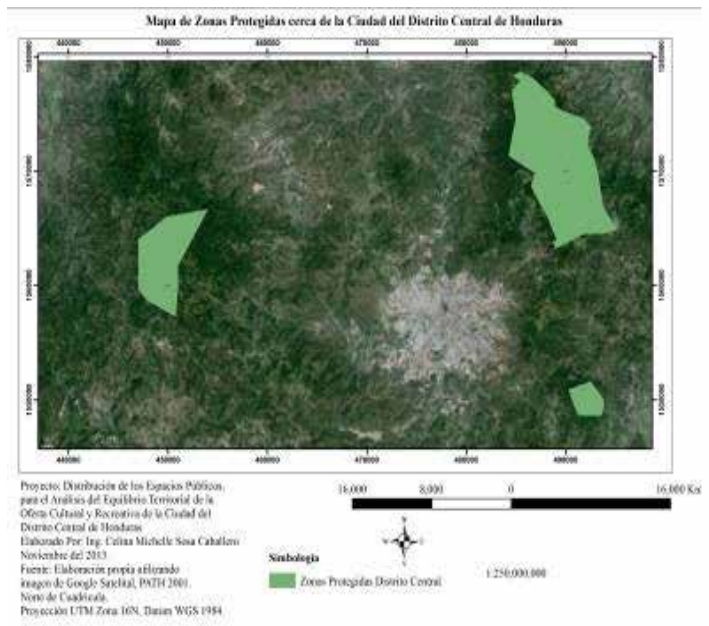

Mapa 8. Zonas Protegidas Cerca de la Ciudad del Distrito Central de Honduras 


\section{Discusión}

De la primera aproximación a las características y distribución espacial de los espacios públicos, de la ciudad del Distrito Central de Honduras, se observa que la mayor parte de las instalaciones corresponden a las de tipo deportiva, donde las de baloncesto y fútbol son mayoría, siendo relevante el dato de la poca dotación de instalaciones de tipo cultural como museos, teatros y parques al aire libre.

Como tendencia general, las instalaciones de baloncesto, fútbol de barrio y las de futbolito, están distribuidas en toda la ciudad y las instalaciones de tipo cultural están concentradas en torno al Centro de la ciudad.

\section{Conclusiones}

En la literatura revisada se habla de que la oferta debe ser diversa y de múltiples expresiones, no obstante en la ciudad del Distrito Central de Honduras no existe esa diversidad o variedad de oferta, debido a que el mayor número de instalación son de carácter deportivo.

Otra premisa de la literatura es sobre la relación más estrecha entre los espacios públicos y los conjuntos habitacionales; en la ciudad del Distrito Central de Honduras esa relación existe principalmente con las canchas de fútbol de las colonias y barrios, y con las canchas de baloncesto.

En esta primera aproximación a la infraestructura cultural y deportiva del Distrito Central se identifican dos tendencias: una, las instalaciones de la oferta cultural (teatros y museos) y, parques y plazas, se localizan en el centro de la ciudad y sus áreas circundantes; la segunda tendencia observada, es que las instalaciones deportivas están dispersas en toda el área urbanizada.

\section{Bibliografía}

- Alcaldía Municipal del Distrito Central Departamento de Deportes; "Informe de Actividades 2010, Presupuesto y Plan de Trabajo - 2011"; Tegucigalpa M. D C; Diciembre de 2010

- $\quad$ AMDC / BID / EGI; Capitulo 6; "Espacios Libres i Equipamiento. Avance Plan Territorial Tegucigalpa”, 2000 - 2015; 2000. 
- Borja, Jordi; Muxí, Zaida; “El espacio público, ciudad y ciudadanía”; Barcelona; http://pensarcontemporaneo.files.wordpress.com/2009/06/el-espacio-publico-ciudad-y-ciudadania-jordi-borja.pdf; 2000.

- Caballero, Lily; Zelaya Marysabel; "Centros Comerciales un Nuevo Espacio de encuentro: Privatización de los Espacios Público o Áreas de Uso Público en lo Privado"; Tegucigalpa, M.D.C., Honduras, C.A: Enero - Abril de 2009

- CCVAH; "Agenda de Ordenamiento Territorial del Concejo Centroamericano de Vivienda y Asentamientos Humanos", San Salvador, El Salvador; 2010.

- $\quad$ Ciudades y Gobiernos Locales Unidos; "Indicadores Culturales y Agenda 21 de la Cultura”; Barcelona; España; 2006.

- Ciudades y Gobiernos Locales Unidos; "Agenda 21 de la Cultura”; Barcelona; España; 2004.

- Congreso Nacional de Honduras; "Ley Marco del Desarrollo Integral de la Juventud”; Honduras; Tegucigalpa; www.inj.gob.hn/Portal/LEY_MARCO_PARA_ EL_DESARROLLO_INTEGRAL_DE_LA_JUVENTUD.pdf; 2006.

- Instituto Nacional de la Juventud; "Política Nacional de Juventud"; Tegucigalpa" Honduras; www.inj.gob.hn/Portal/PNJ.pdf; 2001.

- Instituto Hondureño de Turismo; "Mapa Tegucigalpa Sitios de Interés".

- Organización de Estados Iberoamericanos; Sistema Nacional de Cultura, "Informe Honduras"; http://www.oei.es/cultura2/honduras/cap7.htm\#2.

- PNUD, Capitulo 8; "Un Nuevo Entorno Formal para la Cultura"; En Informe Sobre Desarrollo Humano- Honduras. La Cultura: medio y fin del desarrollo humano http://hdr.undp.org/es/informes/nacional/americalatinacaribe/honduras/honduras_2003_sp.pdf; 2003.

- PNUD; "Hacia la expansión de la ciudadanía"; Informe sobre Desarrollo Humano-Honduras http://hdr.undp.org/en/reports/national/latinamericathecaribbean/honduras/2006_Honduras_web.pdf; 2006. 
- PNUD; "De la Exclusión Social a la Ciudadanía Juvenil”; Informe sobre Desarrollo Humano-Honduras http://hdr.undp.org/es/informes/nacional/americalatinacaribe/honduras/INDH_Honduras_2008-2009.pdf; 2008/2009.

- Secretaria de Cultura Arte y Deporte; "Objetivos Estratégicos"; Honduras; http://www.scad.gob.hn/index.php?option=com_content\&task=view\&id=15\&ltemid $=64 ; 2012$. 